\title{
SUIVI DE LA MIGRATION D'AVALAISON DES ANGUILLES PAR UNE CONDUITE DE DEBIT RESERVE
}

\author{
A. LEGAULT (1), A. ACOU (2), J. GUILLOUËT (1) ET E. FEUNTEUN (2)
}

(1) Fish-Pass, 8 Allée de Guerlédan, Z.A Parc Rocade Sud, 35135 Chantepie, France

(2) UMR 6553 ECOBIO, Université de Rennes 1, 35042 Rennes Cedex, France.

\section{RÉSUMÉ}

Le suivi de la migration catadrome de l'anguille par une conduite de débit réservé a été réalisé sur le barrage de la retenue de Bois Joli sur le Frémur (dénivelé : 13,7 m ; 3 millions de $\mathrm{m}^{3}$ ). Une partie des migrateurs utilise cette conduite, au début de la saison de dévalaison quand elle représente la seule voie de transit mais également plus tard, lorsque le barrage déverse et que s'ouvre ainsi une seconde voie de passage. En raison des faibles débits d'alimentation et de l'implantation de l'entrée de la conduite on peut supposer que les anguilles prospectent la retenue pour trouver une voie de transit. L'efficacité de l'équipement, qui s'établit à $12 \%$ de la dévalaison totale, concourt donc pour une part non négligeable au transit des anguilles. La partition des débits entre les différentes voies de migration semblerait essentielle pour définir leur efficacité. Malgré l'aménagement de la conduite, on observe une mortalité de $11 \%$ des anguilles qui l'empruntent. Elle semble essentiellement liée à l'abrasion contre les parois et le papillon de la vanne. Dans de nombreux cas, la mortalité lors du passage dans les conduites de débit réservé doit être très importante, voire totale en raison de leur configuration et de leur mode de gestion. Cette mortalité tend à réduire le nombre des géniteurs produit par le bassin versant. Les anguilles marquées qui ont franchi le barrage grâce à la conduite avant le pic de migration stoppent leur progression en aval. Elles la reprennent quelques semaines plus tard quand le barrage déverse ou l'année suivante. Ce second constat confirme les effets des retenues sur la vitesse de migration. L'utilisation des conduites de débit réservé par les anguilles d'avalaison devrait être prise en compte lors de leur conception et de leur gestion afin de réduire les risques de mortalité.

Mots-clés : Anguilla anguilla, migration, dévalaison, aménagement.

\section{SURVEY OF DOWNSTREAM MIGRATION OF SILVER EELS THROUGH DISCHARGE PIPE ON A RESERVOIR DAM}

\section{ABSTRACT}

The survey of the catadromous migration of eels through a major dam was operated on a pipe insuring a minimal legal discharge of the dam of Bois Joli on the Frémur River (Northern Brittany, France : height, $13.7 \mathrm{~m}$; Volume, 3 Millions of $\mathrm{m}^{3}$ ). Part of the silver eels used this pipe to emigrate, at the beginning of the downstream run when it represented the only possible downstream passage, but also later when the dam spill enabled a second emigration pathway. Because of the low discharge and of the design of the pipe, it is assumed that eels look for a possible seawards passage. 
Downstream passage through the pipe was estimated to represent $12 \%$ of the downstream run and thus contributes significantly to the transit of silver eels. The contribution of the migration pathways to the migration runs are thought to depend upon the water discharge. Despite its modification to facilitate emigration, the discharge pipe induced the mortality of $11 \%$ of migrating silver eels. Mortality was mainly due to abrasion. In many cases, passage through discharge facilities probably induces high or even total mortality depending upon management options, characteristics of the dam and design of the discharge facility. This induced mortality is likely to reduce significantly the production of breeders by river systems. Marked eels that passed the dam before the emigration peak stopped for a few weeks to one year. This observation confirms that reservoirs decreases migrations speed and efficiency. The dams and other works should be managed in order to facilitate passage and reduce mortality of emigrating silver eels.

Key-words : Anguilla anguilla, silver eel, downstream migration, management.

\section{INTRODUCTION}

Depuis une vingtaine d'années, tous les spécialistes de l'anguille européenne (Anguilla anguilla, L.) constatent une diminution inquiétante des biomasses dans les hydrosystèmes continentaux et côtiers à l'échelle européenne (MORIARTY, 1990). En France, cette baisse des captures est perceptible par l'examen des carnets de pêche (CASTELNAUD et al., 1994) et des inventaires piscicoles (LEGAULT et PORCHER, 1999 ; FEUNTEUN et al., 1999).

Le cycle biologique à la fois long et complexe de cette espèce ne facilite pas l'identification de ce déclin. Cependant, la sur-pêche, la pollution, la régression des habitats continentaux, le parasitisme et l'entrave à la libre circulation par les barrages (TESCH, 1977 ; BRUSLÉ, 1994) sont les facteurs le plus souvent avancés.

Si des études se sont penchées sur la restauration des voies de migration anadrome de l'anguille (LEGAULT, 1994 ; FEUNTEUN et al., 1998), force est de constater que le problème de la migration catadrome des anguilles argentées, les géniteurs potentiels, reste entier.

Lors de sa dernière réunion, le GRISAM (Paimpont, 1998) a exprimé comme principe de gestion de définir des cibles d'échappement de géniteurs en quantité et en qualité. De tels arguments ont été repris dans le rapport de la $11^{\text {ème }}$ réunion du «Working group on eels » qui s'est tenu en septembre 1999 au Danemark (ANON., 1999).

Lors de leur migration catadrome, les anguilles sont confrontées à de nombreux obstacles liés à la multiplication des retenues dans les bassins versants. Les retenues permettent de stocker l'eau pendant les périodes hivernales pour la restituer en période estivale pour l'irrigation ou pour l'adduction d'eau potabilisable. Afin de maintenir l'intégrité des cours d'eau en aval de ces retenues, des débits réservés ou biologiques alimentent la portion aval du bassin versant et ils doivent être légalement au moins égaux au 1/10 du module inter-annuel. Dans les retenues d'alimentation en eau potable, ces débits réservés sont restitués par des conduites. Lors de la phase hivernale de remplissage des retenues, les conduites de débit réservé représentent alors potentiellement les seules voies de transit vers l'aval pour les poissons migrateurs tels que l'anguille en phase de migration catadrome. Ce n'est que plus tard dans la saison que l'ouverture d'autres voies de migration permet également le transit, comme les déversoirs ou les turbines.

Si des études traitant du transit des anguilles argentées dans les turbines hydroélectriques sont disponibles, force est de constater que l'évaluation du transit des 
anguilles par d'autres voies sont rares, en particulier l'utilisation des conduites de débit réservé des retenues. Les aménagements réalisés sur le bassin versant du Frémur donnent l'opportunité de disposer d'un site propice à une telle étude qui permettra d'évaluer l'utilisation de ces conduites pour la migration catadrome, de préciser leur efficacité et de décrire les possibilités d'améliorer le transit par ces aménagements.

\section{MATÉRIELS ET MÉTHODES}

\section{Présentation du site}

Le barrage du Bois Joli crée une retenue pour l'alimentation en eau potable de la région de Dinard et Saint Malo. Elle est alimentée par le bassin versant du Frémur qui s'étend sur $50 \mathrm{~km}^{2}$ en amont du barrage. Elle permet de stocker l'eau pendant la période hivernale afin d'assurer l'alimentation en eau potable de cette région touristique pendant la période estivale.

L'ouvrage comporte une crête déversante à la cote 28,20 m NGF. Avec un niveau d'eau aval de 14,70 m NGF, la chute maximale atteint 13,50 m. Lorsque la retenue est pleine, elle s'étend sur 59 hectares pour un volume de $3000000 \mathrm{~m}^{3}$.

La gestion du barrage permet de maintenir un débit réservé de $0,04 \mathrm{~m}^{3} / \mathrm{s}$ sur la portion aval du bassin versant. Lors de la période de remplissage puis d'utilisation de la retenue, la portion aval de la rivière est uniquement alimentée par le débit réservé. Quand la retenue est pleine, elle est également alimentée par le débit naturel du bassin versant qui transite par la crête déversante.

La conduite de débit réservé est constituée d'une canalisation de $200 \mathrm{~mm}$ de diamètre qui est branchée sur une canalisation de $800 \mathrm{~mm}$ de diamètre, elle même située à la sortie de la tour de prise d'eau. Cette dernière est munie de 5 vannes qui permettent de choisir le niveau de prise d'eau dans la retenue. Seule la vanne médiane d'une section de $0,5 \mathrm{~m}^{2}$ à la cote $21,00 \mathrm{~m} \mathrm{NGF}$ est ouverte pendant l'étude. La canalisation de débit réservé est équipée d'une vanne papillon qui permet de régler le débit réservé en fonction du niveau de la retenue. Quand la retenue est pleine, la largeur maximale de la section libre de la vanne atteint $2 \mathrm{~cm}$. Des tronçons et des cadavres d'anguilles sont régulièrement observés lors des manœuvres des vannes.

La retenue de Pont es Omnès est localisée en aval immédiat du barrage de Bois Joli. Elle est équipée d'une station de contrôle des migrations anadrome et catadrome située à $1 \mathrm{~km}$ en aval du barrage de Bois Joli. La station fait l'objet d'un suivi en continu depuis 1996.

\section{Dispositif expérimental}

Suite aux observations de mortalité à la sortie de la conduite de débit réservé, celle ci a été modifiée. Une nouvelle section de contrôle du débit est installée en aval de la vanne papillon, son diamètre intérieur s'établit à $75 \mathrm{~mm}$ (Figures 1et 2) et permet le transit des anguilles. La vanne est laissée grande ouverte. La conduite de débit réservé débouche sous le niveau d'eau aval à la cote 13,70 m. Désormais, le débit de la canalisation varie avec le niveau de la retenue entre 0,042 et $0,048 \mathrm{~m}^{3} / \mathrm{s}$. Un filet est installé au débouché de la réduction : longueur : $6,0 \mathrm{~m}, \varnothing: 0,50 \mathrm{~m}$, maille : $2 \mathrm{~mm}$ (Figure 3). II permet de capturer les animaux en transit par la canalisation.

\section{Suivi du système de piégeage}

Le piégeage débute le 15 octobre 1998 et s'achève le 18 janvier 1999 en raison de la détérioration du filet de capture. Le rythme des relèves est régulier pendant toute 
la phase de l'étude (approximativement 3 relèves par semaine). Ainsi, 40 relèves sont effectuées. Les données biométriques individuelles sont recueillies sur l'ensemble des poissons capturés ainsi que leur état sanitaire. Les anguilles vivantes sont ensuite stockées dans un vivier au minimum pendant trois jours pour observer une éventuelle mortalité différée. Elles sont ensuite relâchées après marquage éventuel à l'aide de PITtags (PRENTICE et al., 1990a ; PRENTICE et al., 1990b) en aval du barrage. Ainsi, 38 anguilles sont marquées avant d'être relâchées en aval du barrage du Bois Joli entre le 22 octobre et le 2 décembre 1998.

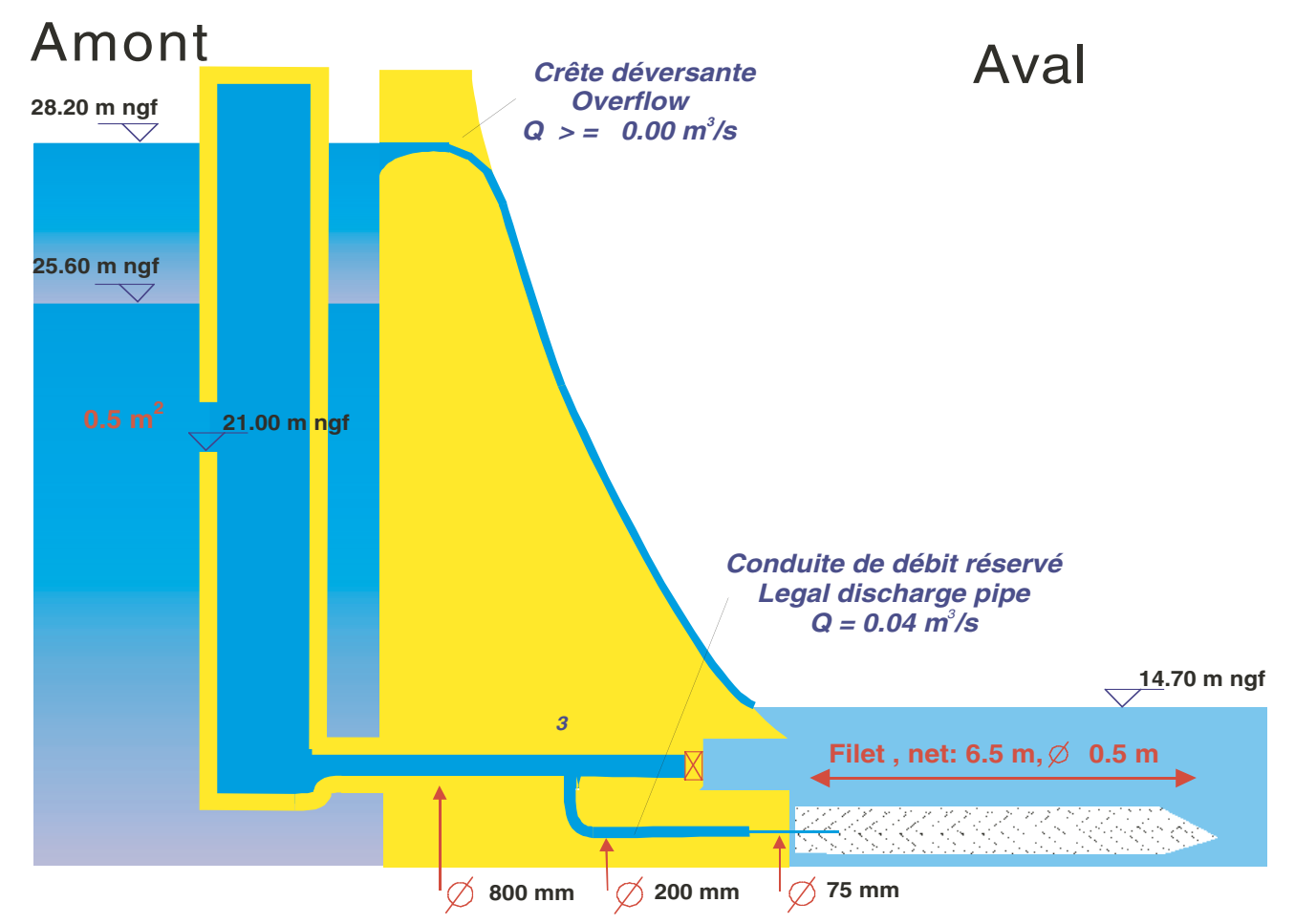

\section{Figure 1}

Schéma du site expérimental.

\section{Figure 1}

Configuration of the experimental site.

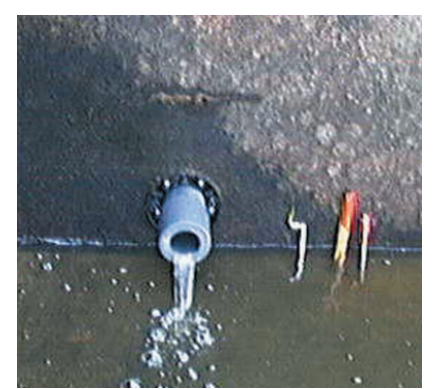

Figure 2

Tube de réduction de débit ajouté à la sortie de la conduite de débit réservé.

\section{Figure 2}

Discharge pipe modified to facilitate passage of eels.

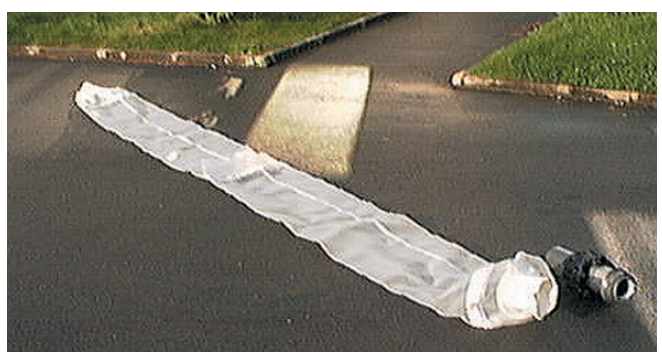

Figure 3

Filet de capture installé à la sortie de la conduite.

\section{Figure 3}

Gear used to sample eels at the end of the discharge pipe. 
Le suivi de la migration est réalisé simultanément en aval sur le piége de Pont es Omnès avec recueil des données biométriques et contrôle du marquage. Le stade de maturation est également déterminé suivant la méthode utilisée précédemment sur ce cours d'eau (FEUNTEUN et al., 2000). Les anguilles sont considérées comme argentées lorsque au moins deux des trois critères suivants sont observés : présence d'une ligne latérale pigmentée, couleur noire sur le dos et argentée sur le ventre et index oculaire de Pankhurst (1982) > 6,5.

Les données de niveau de la retenue sont enregistrées. Les données hydrologiques sont acquises grâce à une station de jaugeage (SEMA de Bretagne) située à l'amont immédiat de la retenue.

\section{RÉSULTATS}

\section{Évolution du niveau d'eau de la retenue}

Lors de la phase de remplissage hivernal, le niveau de la retenue augmente en fonction du débit naturel (Figure 4). La durée de la phase de remplissage varie selon les conditions hydrologiques annuelles. Une fois la retenue pleine, l'eau est évacuée par le déversoir (Figure 1).

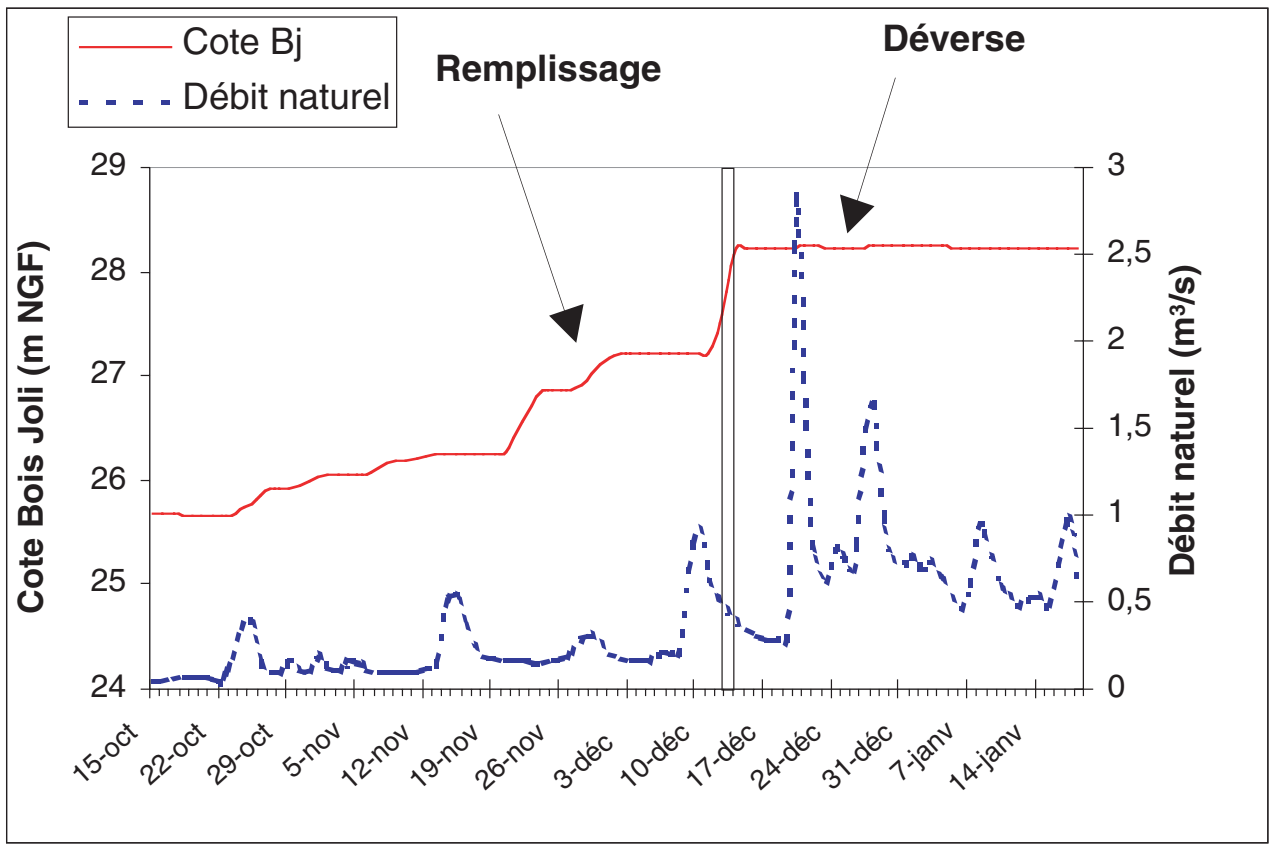

\section{Figure 4}

Variations de la cote de la retenue du Bois Joli et du débit naturel en hiver 1998/99.

\section{Figure 4}

\section{Variations of the water level and the natural water flow at Bois Joli during} 1998/1999 winter.

Au début de l'étude, le 15 octobre, le niveau de la retenue s'établit à 25,60 m NGF. La retenue déverse à partir du 14 décembre 1998 (28,20 m NGF). Ainsi, pendant la phase de remplissage, jusqu'au 14 décembre 1998, seul le débit réservé $\left(0,04 \mathrm{~m}^{3} / \mathrm{sec}\right)$ alimente la portion aval du bassin versant. Par la suite, le débit est essentiellement évacué par le seuil déversant du barrage mais également par la conduite de débit réservé. La station de jaugeage permet de caractériser les fluctuations de débits. 


\section{Les captures}

\section{Les espèces observées}

Seules trois espèces sont capturées dans le filet :

$\begin{array}{lr}\text { Anguilles (Anguilla anguilla) : } & 105 \text { individus } \\ \text { Perches (Perca fluviatilis) : } & 25 \text { individus } \\ \text { Gardons (Rutilus rutilus) : } & 8 \text { individus }\end{array}$

Les anguilles représentent donc $76 \%$ des captures totales.

\section{Les anguilles capturées}

Au total, 105 anguilles sont capturées pendant la période du suivi, dont 101 anguilles argentées et 4 anguilles jaunes. Les anguilles jaunes ont des tailles inférieures à $181 \mathrm{~mm}$, elles sont écartées de l'analyse.

La gamme de taille des anguilles argentées est large, elle varie entre 313 et $772 \mathrm{~mm}$. (Figure 5) Toutefois, la majorité des anguilles ont des tailles inférieures à $440 \mathrm{~mm}$. L'identification du sexe des anguilles argentées a été réalisée précédemment sur un échantillon de 39 individus (ACOU, 1999). En raison du dimorphisme sexuel important qui existe chez les anguilles (KUSHNIROV et DEGANI, 1995), les mâles présentent des tailles inférieures à $440 \mathrm{~mm}$.

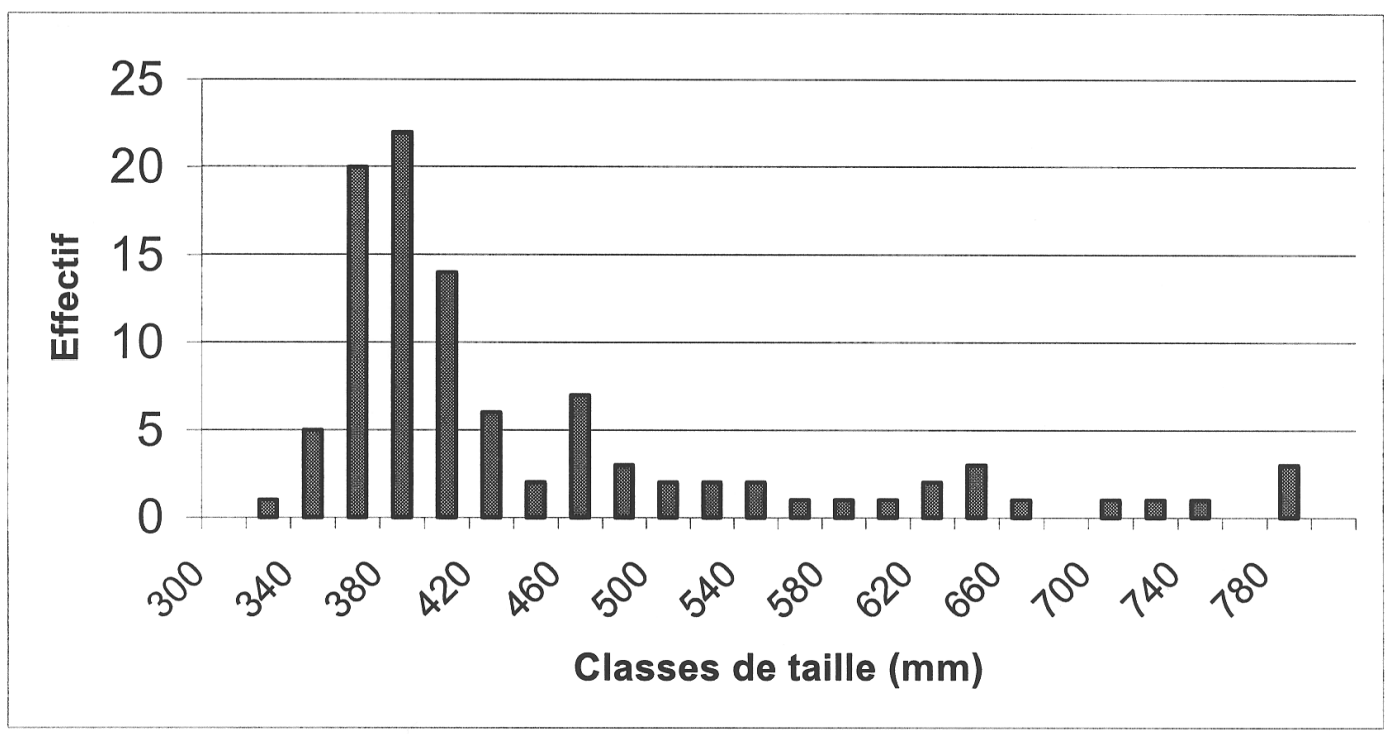

Figure 5

Distribution des tailles des anguilles argentées capturées.

\section{Figure 5}

Length distribution of the sampled silver eels.

\section{Mortalité lors du transit}

11 anguilles argentées sont mortes après le transit. La mortalité globale affecte $10,9 \%(\mathrm{~N}=11 / 101)$ des anguilles en transit. Deux types de mortalité peuvent être distingués : 
- La mortalité « instantanée », observée à l'occasion de la relève du filet, est la plus importante, elle concerne 10 anguilles argentées.

- La mortalité différée, estimée après stabulation dans un bac des anguilles pendant 3 jours, ne concerne qu'un seul individu.

En raison des faibles effectifs, il est difficile de caractériser la mortalité différentielle en fonction de la taille des anguilles. Toutefois le taux de mortalité semble plus faible pour les petits individus 7,7\% (5/65) taille < $440 \mathrm{~mm}$ et $24 \%(6 / 25)$ (i.e. $>440 \mathrm{~mm}$ ).

Les anguilles mortes ont un épiderme à vif et l'on note une disparition importante du mucus (Figure 6).

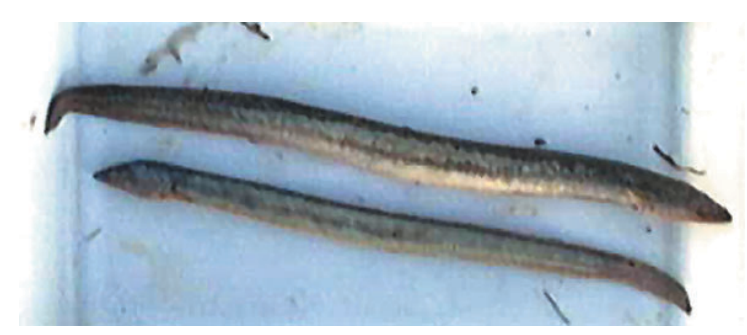

\section{Figure 6}

Anguilles capturées mortes (Des marques d'abrasion sont situées sur les flans).

\section{Figure 6}

Eels killed during their passage (Abrasion marks are visible on the sides).

\section{Rythme de transit}

Le piégeage des anguilles argentées commence le 15 octobre 1998 pour s'achever au début du mois de janvier 1999. Deux phases peuvent être distinguées (Figure 7) :

La phase de remplissage jusqu'au 13 décembre 1998, le transit des anguilles s'effectue uniquement par la conduite de débit réservé, 53 anguilles argentées l'ont empruntée.

La phase de déversement qui ouvre une seconde voie de migration par le seuil déversant à partir du 14 décembre, seule une faible proportion du débit transite par la conduite (moins de $4 \%$ du débit naturel), 48 anguilles argentées ont emprunté la conduite lors de cette phase.

Quelle que soit la période de migration, on observe des fluctuations importantes de l'intensité migratoire. Le rythme des relèves du filet ne permet pas de décrire la relation des captures avec le débit journalier. Avant le 14 décembre 1998, la relation entre les captures d'anguilles argentées et le débit est difficile à mettre en évidence. Toutefois, le premier pic de capture correspond à la première augmentation notable du débit amont. 


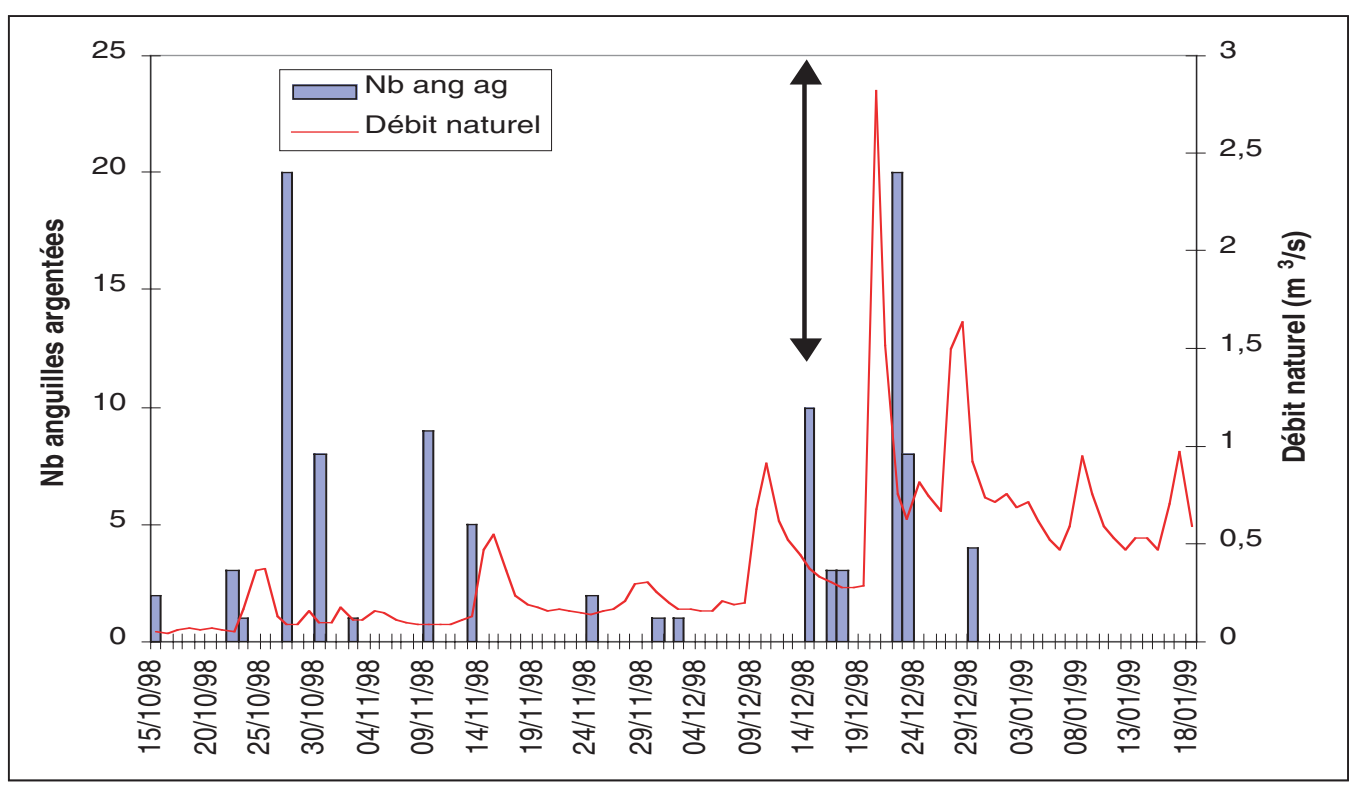

Figure 7

Évolution du transit d'anguilles et du débit naturel (flèche : début de déversement).

\section{Figure 7}

Temporal variations of the number of migrating eels and of the water flow (arrow ; overflow beginning).

\section{Re-captures des anguilles marquées}

Avant la phase de déversement, 38 anguilles ont été marquées et relâchées en aval du barrage du Bois Joli. Au total, 10 individus, soit environ $26 \%$ ont été re-capturés au piège de Pont es Omnès ; 8 entre le 16 et le 20 décembre 1998, 2 en 1999 et 0 en 2000.

Les anguilles ont été re-capturées entre 18 et 398 jours après leur lâcher (Tableau 1). En 1998, elles ont été reprises après la phase de débordement, soit après une stabulation entre 18 et 56 jours dans la retenue lors du pic de migration1998. En 1999, elles ont été re-capturées avant le débordement et avant le pic annuel de migration 1999.

\section{Tableau 1}

\section{Marquage/re-capture}

\section{Tableau 1}

Results of mark recapture surveys.

\begin{tabular}{|c|c|c|c|}
\hline $\mathrm{N}^{\circ}$ & Date de lâcher & Date de re-capture & Nombre de Jours \\
\hline 1 & $30 / 10 / 98$ & $16 / 12 / 98$ & 47 \\
\hline 2 & $27 / 10 / 98$ & $19 / 12 / 98$ & 53 \\
\hline 3 & $22 / 10 / 98$ & $17 / 12 / 98$ & 56 \\
\hline 4 & $27 / 10 / 98$ & $16 / 12 / 98$ & 50 \\
\hline 5 & $30 / 10 / 98$ & $17 / 12 / 98$ & 48 \\
\hline 6 & $27 / 10 / 98$ & $17 / 12 / 98$ & 51 \\
\hline 7 & $30 / 10 / 98$ & $17 / 12 / 98$ & 48 \\
\hline 8 & $02 / 12 / 98$ & $20 / 12 / 98$ & 18 \\
\hline 9 & $27 / 10 / 98$ & $05 / 10 / 99$ & 342 \\
\hline 10 & $09 / 11 / 98$ & $12 / 12 / 99$ & 398 \\
\hline
\end{tabular}




\section{DISCUSSION}

\section{Utilisation de la conduite de débit réservé comme voie de migration}

Ce suivi a permis de caractériser l'utilisation des conduites de débit réservé par l'anguille lors de sa migration catadrome. Le débit réservé s'établit à environ $0,04 \mathrm{~m}^{3} / \mathrm{sec}$. Initialement, en période d'étiage, il correspond au débit naturel. Puis le débit de remplissage augmente et la proportion du débit évacuée diminue. Durant ces périodes la conduite représente la seule voie de passage et des anguilles l'ont trouvée. Ultérieurement, quand la retenue est pleine, le débit est évacué par la conduite mais surtout par le déversoir, certaines anguilles transitent encore par la conduite. Les anguilles ont donc trouvé ce passage dont l'entrée se situe entre 4,50 et de 7,20 mètres de profondeur et dont la vitesse de courant à l'entrée est inférieure à $0,10 \mathrm{~m} / \mathrm{s}$.

Ces observations semblent donc caractériser une recherche des voies de migration, au moins pour une partie des anguilles. Elles utilisent toutes les zones d'évacuation de débit pour franchir l'obstacle lors de la migration catadrome. Si les zones de transit ne sont pas adaptées au passage des animaux, ceux qui s'y engagent risquent d'y être tués ou blessés.

\section{Efficacité du transit par la conduite}

L'efficacité de la conduite peut être définie comme le rapport du nombre d'anguilles empruntant la conduite de débit réservé sur le nombre total d'anguilles transitant par l'ouvrage. Le transit par le seuil déversant n'étant pas connu, il doit être estimé. Deux approches distinctes peuvent être utilisées.

\section{Efficacité par rapport au suivi du piège aval}

Le suivi du piège de dévalaison du Pont es Omnès (situé $1 \mathrm{~km}$ en aval) permet d'évaluer le nombre d'anguilles en migration. Pour la saison 1998, elle est estimée à 819 individus (FEUNTEUN et al., 2000 ; GUILLOUËT et al, 2000). La conduite aurait contribué au transit de $12,3 \%$ de la population migrante Toutefois, cette estimation n'intègre pas la mortalité lors du passage dans la conduite de débit réservé du barrage de Bois Joli et l'efficacité pourrait être surestimée.

\section{Évaluation par rapport au volume filtré}

Si l'on fait hypothèse initiale que le transit d'anguilles argentées dans la conduite est proportionnel au débit évacué, le nombre de migrants sur l'ouvrage du Bois Joli peut être estimé grâce aux données de débit enregistrées à la station de jaugeage et aux densités estimées grâce au filet de la conduite. Pendant la phase de pré-déversement de la retenue, toutes les anguilles transitent par la conduite. Lorsque l'ouvrage déverse, le nombre d'anguilles en transit par chaque ouvrage est estimé au prorata du débit de chacun d'entre eux

Sur cette base, le nombre d'anguilles migrantes est estimé à 857 individus. La contribution de la canalisation s'élève donc à 11,8\%. L'estimation est légèrement supérieure aux données enregistrées au piège de Pont es Omnès.

La conduite de débit réservé permet le transit d'environ 3 à $12 \%$ de la population migrante. II a lieu avant et après le déversement. En outre, la seconde estimation pourrait indiquer le rôle prépondérant du débit sur l'efficacité de la conduite à l'échelle saisonnière. Ces résultats sont conformes à ceux de ACOU (1999) qui montrent que le débit est le facteur principal qui explique la variabilité de l'intensité migratoire au cours des pics de migration. 
Ces résultats plaident pour l'intérêt d'une gestion spécifique des conduites de débit réservé. En effet, ils indiqueraient que le débit en transit est l'un des facteurs essentiel qui régit l'efficacité des aménagements de dévalaison pour l'anguille. Ainsi, des mesures de gestion adaptées permettraient d'améliorer les conditions de franchissement par l'utilisation de vannages dans l'attente de la mise au point d'équipements spécifiques de dévalaison.

\section{Tableau 2}

Évaluation du transit des anguilles au barrage du Bois Joli.

\section{Tableau 2}

Estimation of the eel passages over or through the dam of Bois Joli.

\begin{tabular}{|c|c|c|r|}
\hline & Conduite & Crête déversante & \\
\hline Période & Nb ang. capturées & Nb ang. estimé & Total \\
\hline $1 / 10 / 98$ au $13 / 12 / 98$ & 53 & 0 & 53 \\
\hline $14 / 12 / 98$ au $18 / 01 / 99$ & 48 & 756 & 804 \\
\hline Total & 101 & 756 & 857 \\
\hline
\end{tabular}

\section{La mortalité des anguilles lors du transit dans la conduite}

Une partie des anguilles argentées migrantes, géniteurs potentiels, emprunte la canalisation de débit réservé. Malgré les aménagements apportés, il subsiste une mortalité résiduelle d'environ $11 \%$. Ces résultats semblent caractériser une mortalité liée à l'abrasion des animaux contre les parois de la conduite.

La configuration de la conduite permet de distinguer plusieurs zones critiques qui engendrent des variations brutales de vitesse du courant et de direction.

Les changements de section des conduites (Figure 1) provoquent une augmentation rapide de la vitesse. Entre les sections 800 et $200 \mathrm{~mm}$, la vitesse observée passe ainsi de moins de $0,10 \mathrm{~m} / \mathrm{s}$ à plus de $1,20 \mathrm{~m} / \mathrm{s}$ pour atteindre plus de $9,00 \mathrm{~m} / \mathrm{s}$ dans la conduite de $75 \mathrm{~mm}$.

La canalisation est constituée de tuyaux soudés, deux zones à risques peuvent ainsi être définies sur la canalisation de $200 \mathrm{~mm}$. A sa base, elle est soudée à $90^{\circ}$ sur la canalisation de $800 \mathrm{~mm}$ puis, dans sa partie médiane, la jonction de deux tuyaux placés une nouvelle fois à $90^{\circ}$ assure le changement d'orientation de la canalisation. En outre, malgré son ouverture complète, la vanne en position centrale risque également d'être agressive.

La configuration de la canalisation de débit réservé apparaît agressive pour l'anguille et responsable des mortalités observées lors du transit. Ces mortalités devaient être encore plus importantes voire totales avant les aménagements apportés au début de l'étude. Cependant, la mortalité de $11 \%$ enregistrée à la sortie de la conduite de débit réservé aurait pu être réduite par un dispositif conçu dès la construction du barrage pour concilier dévalaison des anguilles et restitution du débit réservé. II apparaît donc essentiel d'intégrer les possibilités de migration catadrome lors de la conception des conduites de débit réservé afin de réduire les risques de mortalité des anguilles argentées. En particulier il faudrait éviter les sections de contrôle de débit réservé trop étroites. De plus, la gestion hydraulique de ces ouvrages devrait être modulée pendant les périodes de migration afin de limiter la mortalité. En particulier, lors des périodes de fort débit favorable à la migration catadrome, les vannes des conduites devraient être grandes ouvertes. 


\section{Figure 8}

Autre exemple de mortalité d'Anguille dans une conduite de débit réservé.

\section{Figure 8}

\section{Another example of eel mortality in a by-pass.}

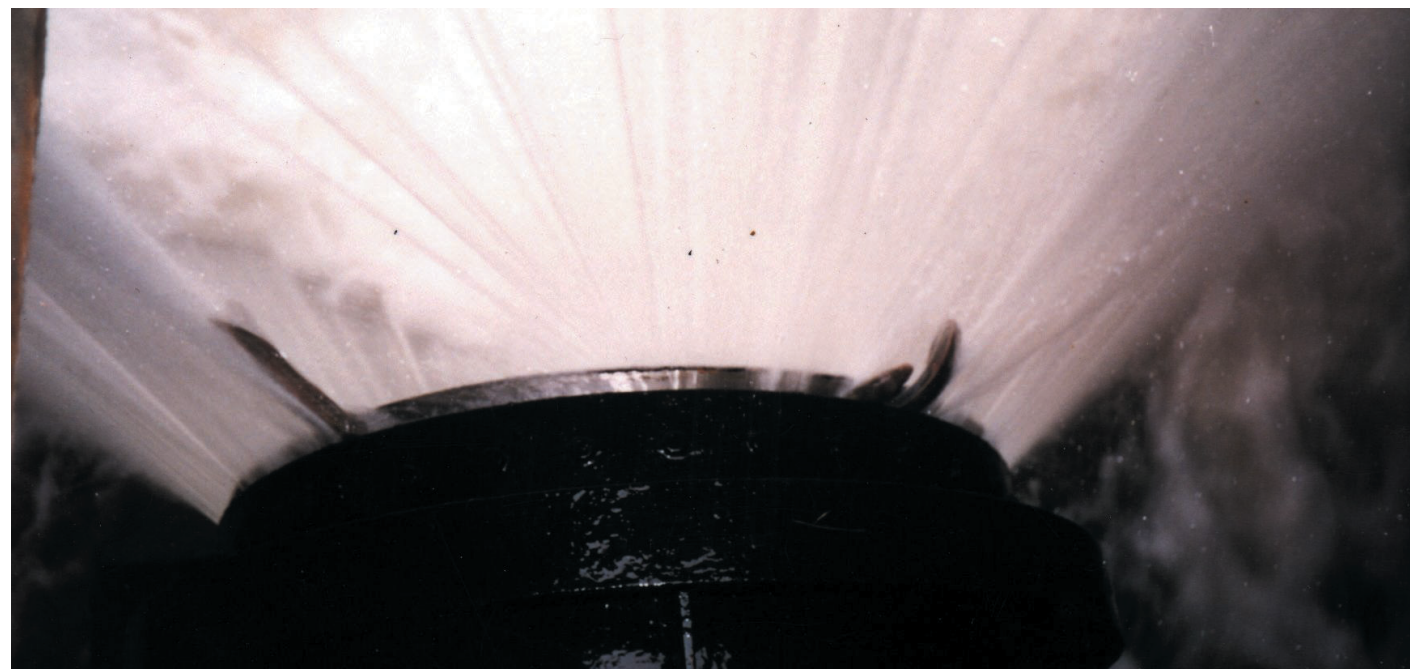

\section{Suivi des migrateurs en aval de la retenue}

Les expériences de marquage - re-capture d'anguilles entre les sites du Bois Joli et du Pont es Omnès apportent également quelques éléments sur la dynamique migratoire des anguilles argentées. En 1998, la durée de transit s'établit entre 18 et 56 jours alors que la distance entre les deux sites représente moins d'un kilomètre. La retenue provoque une artificialisation du régime hydraulique. Pendant la phase initiale de remplissage, seul le débit réservé alimente la portion aval du bassin versant. Les anguilles ayant transité par l'ouvrage amont s'accumulent dans la retenue aval, elles reprennent leur migration à l'occasion de l'augmentation de débit. Elles sont alors toutes re-capturées dans un délai de 4 jours. Toutefois, d'autres individus attendent une année avant de reprendre leur migration catadrome. Ces données confirment les observations de VOLLESTADT et al. (1986) sur les possibilités d'arrêt de la migration, et l'analyse des dynamiques migratoires sur ce bassin versant, montrant que près de $3 \%$ des anguilles présentes dans le cours d'eau migrent une à trois années suivant leur argenture (FEUNTEUN et al., 2000).

Les retenues seraient donc responsables d'un retard de migration en liaison avec les modifications de régime hydraulique qu'elles engendrent. Elles auraient également pour conséquence de concentrer les périodes de migration. Elles influenceraient donc également la migration catadrome sur la portion aval du bassin versant. En outre, le passage au travers des conduites de débit réservé, ou par dessus un barrage de cette importance, représentent un stress pouvant provoquer un arrêt migratoire définitif ou temporaire des anguilles qui réduirait encore le potentiel de production de géniteurs des bassins versants ainsi équipés.

\section{CONCLUSION}

Les conduites de débit réservé sont utilisées par l'anguille lors de sa migration catadrome. Toutefois leur configuration et leur gestion peuvent entraîner la mortalité des migrants. Des modalités de gestion et une configuration adaptée pourraient améliorer la situation actuelle dans l'attente de la mise au point d'ouvrages de dévalaison ou d'une gestion spécifique pour cette espèce. 


\section{REMERCIEMENTS}

Cette étude a été menée en complément des travaux réalisés sur le Bassin du Frémur grâce au soutien de la FDAAPPMA d'Ille et Vilaine et de la DIREN de Bretagne.

\section{BIBLIOGRAPHIE}

ACOU A., 1999. L'anguille européenne (Anguilla anguilla, L. 1758) : Dynamique et déterminisme de la migration catadrome dans un bassin versant anthropisé de Bretagne (Frémur). Diplôme Doctoral de Recherche de l'Université de Rennes 1-167 pp. + annexes.

BRUSLE J., 1994. L'Anguille européenne Anguilla anguilla, un poisson sensible aux stress environnementaux et vulnérable à diverses atteintes pathogènes. Bull. Fr. Pêche Piscic. 335 : 237-260.

CASTELNAUD G., GUERAULT D., DESAUNAY Y. et ELIE P., 1994. Production et abondance de la civelle en France au début des années 90. Bull. Fr. Pêche et Piscic., vol. 335 : p. 263-288.

FEUNTEUN E., ACOU A., GUILLOUËT J., LAFFAILLE P. et LEGAULT A., 1998. Spatial distribution of an eel population (Anguilla anguilla, L.) in a small catchment of northern Brittany (France) - Consequences of hydraulic works. Bull. Fr. Pêche et Piscic., 349 : 129-139.

FEUNTEUN E., C. RIGAUD, P. ELIE et JC LEFEUVRE 1999. Les peuplements piscicoles des marais littoraux de l'ouest de la France : un patrimoine à gérer ? Bull. Fr. Pêche et Piscic. 352 : 63-79.

FEUNTEUN E., ACOU A., LAFFAILLE P. et LEGAULT A., 2000. The European Eel (Anguilla anguilla, L.): prediction of spawner escapement from continental population parameters, Can. J. Fish. Aquat. Sci. 57 : 1627-1635.

GUILLOUËT J., ACOU A., MOUNAIX B., LEGAULT A., FEUNTEUN E., 2000. Etude de la restauration de la population d'anguille sur le Frémur : synthèse du suivi de 1995 à 1999., rapport Fish-Pass, 104 p.

LANGON M. et DARTIGUELONGUE J., 1996. La Dévalaison des anguilles (Anguilla anguilla L.) argentées - Test de deux dispositifs de dévalaison à la centrale hyroélectrique EDF d'Halsou (Nive, Pyrénées-Atlantiques), 1996 - Revue Bibliographique. Rapport de sous-traitance INRA/SCEA, 110 pp. + annexes.

LEGAULT A., 1994. Etude préliminaire du recrutement fluvial de l'anguille. Bull. Fr. Pêche Piscic. 335 : 33-41.

LEGAULT A., PORCHER J.P., 1999 : Eels populations and recent evolution on the Orne river, Normandy, France. communication REUNION EIFAC 2000

MORIARTY C., 1990. European catches of elver of 1928-1988. Int. Revue ges. Hydrobiol., 75, 701-706.

TESCH F.-W., 1977. The eel. Biology and management of anguillid eels. London, Chapman et Hall. 434 p.

VOLLESTAD L.A., B. JONSSON, N.A. HVIDSTEN, T.F. NAESJE, Ø. HARALDSTAD and J. RUUD-HANSEN, 1986. Environmental factors regulating the seaward migration of European silver eels (Anguilla anguilla). Can. J. Fish. Aquat. Sci. 43 : 1909-1916. 